\title{
Maintenance Chemotherapy in Acute Promyelocytic Leukemia: The Thin Line Between Survival and Immunosuppression
}

\author{
Sita Bhella ${ }^{1}$, Catherine Maurice ${ }^{2 *}$, Lili-Naz Hazrati ${ }^{3}$, Robert Chen ${ }^{4}$, \\ Andre C Schuh ${ }^{5}$, Warren P Mason ${ }^{2}$, Austin M Pereira ${ }^{6}$, Amanda \\ Pereira $^{7}$ and Matthew Seftel ${ }^{5}$ \\ ${ }^{1}$ Department of Hematology, Queen's University, Kingston, Canada \\ ${ }^{2}$ Department of Medicine, Division of Neurology, Princess Margaret Cancer Centre, \\ Toronto, Canada \\ ${ }^{3}$ Paediatric Laboratory Medicine, Hospital for Sick Children, Toronto, Canada \\ ${ }^{4}$ Department of Medicine, Division of Neurology, Toronto Western Hospital, Toronto, \\ Canada \\ ${ }^{5}$ Department of Medicine, Division of Hematology-Oncology, Princess Margaret \\ Cancer Centre, Toronto, Canada \\ ${ }^{6}$ Faculty of Medicine, University of Toronto, Toronto, Canada \\ ${ }^{7}$ Faculty of Medicine, Saba University, Dutch Caribbean \\ *Corresponding Author: Catherine Maurice, Department of Medicine, Division of \\ Neurology, Princess Margaret Cancer Centre, Toronto, Canada.
}

DOI: $10.31080 /$ ASCB.2020.04.0205
Received: December 09, 2019

Published: January 29, 2020

(C) All rights are reserved by Catherine

Maurice., et al.

\begin{abstract}
We describe a case of progressive multifocal leukoencephalopathy (PML) in a patient on maintenance chemotherapy for high-risk acute promyelocytic leukemia (APL). The highlight of this scenario is the clinical improvement of the PML after the cessation of maintenance chemotherapy. We demonstrate that profound immunosuppression can occur during maintenance chemotherapy for APL. The role of post-consolidation maintenance chemotherapy in APL is controversial and previous studies demonstrated an improved disease-free survival with observation versus maintenance chemotherapy for APL. A phase 3 study by Lo Coco., et al. (2013) concluded that ATRA plus ATO with no maintenance chemotherapy was not inferior to standard chemotherapy. This case emphasizes that maintenance chemotherapy is associated with infectious risks the role for maintenance chemotherapy in APL should be re-evaluated. Keywords: PML Progressive Multifocal Leukoencephalopathy; JC Virus; Leukemia; ATRA; Maintenance Chemotherapy; ATO (Arsenic Trioxide); Immunosuppression; Myoclonus
\end{abstract}

Key points

- $\quad$ Post-consolidation maintenance chemotherapy is a subject of controversy in APL and can lead to life threatening immunosuppression.

- Iatrogenic development of progressive multifocal leukoencephalopathy is treatable if diagnosed promptly, awareness is crucial.

\section{Introduction}

Progressive multifocal leukoencephalopathy (PML) is an opportunistic infection historically associated exclusively with a concomitant HIV infection. Immunosuppression is the major risk factor and nowadays, lymphoreticular malignancies represent the most common non-AIDS etiology. When drugs are implicated, a prompt 
diagnosis, the interruption of the causing agent and a rapid restoration of the baseline immune function could lead to survival, and quasi-complete recovery is possible. We report a case describing the dilemma between the wish to consolidate optimally the recovery, and the risks related to this approach.

\section{Case Report}

A 54 year-old woman with high risk PML-RARA positive acute promyelocytic leukemia (APL) in hematologic and molecular remission for two years presented with progressive myoclonus and right leg weakness. She had previously received remission induction chemotherapy with tretinoin (ATRA), daunorubicin, cytarabine, and consolidation cycles with ATRA and high dose cytarabine, followed by nineteen months of maintenance chemotherapy with intermittent ATRA, methotrexate and 6-mercaptopurine. Over the course of the next six months, she developed progressive bilateral lower limb weakness, spasticity, myoclonus and ataxia.

Repeat marrow aspirate demonstrated ongoing morphological and molecular remission and her absolute lymphocyte count (ALC) was $0.10 \mathrm{x} 109 / \mathrm{L}$. Contrast-enhanced brain and spine MRIs were unremarkable. A first lumbar puncture was negative for malignancy and showed normal chemistry and cell counts. A second enhanced MRI of the nervous system showed interval development of confluent symmetric white matter changes in the subcortical white matter of bilateral precentral gyri and multifocal patchy enhancement, without involvement of the cervical spine (Figure 1). A complete work-up including two supplemental lumbar punctures, serum vitamin B12 level, TSH, ANA, HTLV-1 and 2 antibodies, HIV 1/ 2 antibodies, HIV P24 antigen, CMV serum PCR, and anti-deaminated gliadin antibody were negative. The PCR for JC virus was negative in the CSF. A right frontal biopsy of the lesion located in motor cortex showed the presence of SV-40 immunoreactive oligodendrocytes, consistent with a diagnosis of Progressive Multifocal Leukoencephalopathy (PML) (Figure 2).

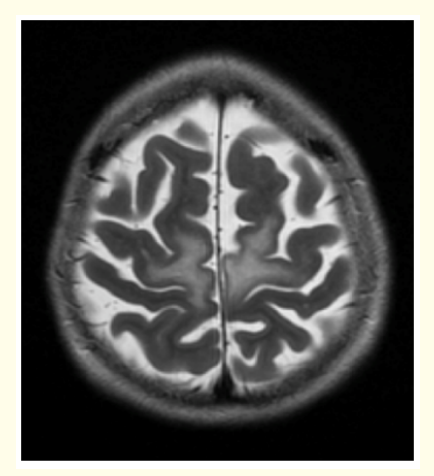

Figure 1: Brain MRI, axial T2 sequence, showing nearly symmetrical white matter lesions within the paracentral lobules.

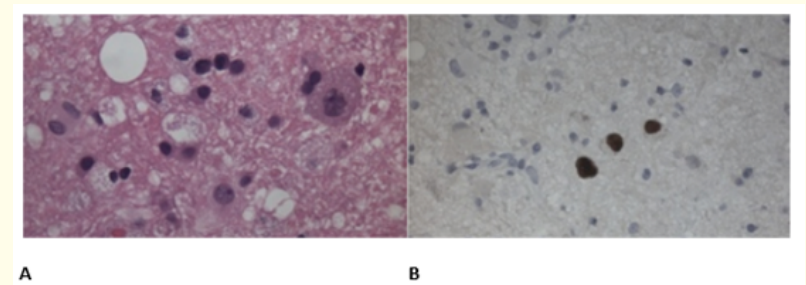

Figure 2: Brain Biopsy (scale bar $250 \mu \mathrm{m}$ ): (A) Brain biopsy centered on the white matter with increased inflammation (lymphocytes and macrophages). The oligodendroglial nuclei have a glassy appearance.

Brain Biopsy (scale bar $250 \mu \mathrm{m}$ ): (B) Immunohistochemistry shoes positivity of SV-40 in the oligodendroglial nuclei, confirming the diagnosis of PML.

Maintenance chemotherapy was stopped five months after the onset of neurological symptoms. Despite this, she developed progressive incapacitating myoclonus, dysphagia and became bed-ridden. She was treated palliatively. However, six months after maintenance chemotherapy was stopped, she was able to communicate verbally, and her strength and myoclonus improved. Her ALC improved to $1.80 \times 109 / \mathrm{L}$. MRI performed nine months after clinical improvement demonstrated a significant reduction in the size of the subcortical lesions and complete resolution of the enhancement, suggesting decreased disease activity.

\section{Discussion}

PML results from an infection of the oligodendrocytes by JC virus, within the subcortical white matter, often lethal or resulting in permanent neurologic deficits. JC virus is acquired during childhood or young adulthood with a prevalence of 50-70\%1. Immunosuppression is a key feature leading to viral reactivation from an extra-neural primary site, involvement of the central nervous system and development of neurologic deficits reflecting the brain areas involved [1]. Concomitant HIV infection is the leading risk factor, but lymphoproliferative malignancies and the use of immunosuppressive drugs are all potential triggers of PML reactivation $[2,3]$. JC virus may be negative in the CSF by quantitative PCR in a patient with PML proven by biopsy. Weber., et al. estimated that the sensitivity JC virus by quantitative PCR was only 75\% [4].

\section{Conclusion}

This case demonstrates that profound and clinically significant immunosuppression, as measured by the patient's lymphopenia, can occur during maintenance chemotherapy for APL. The role of post-consolidation maintenance systemic chemotherapy in APL is controversial. Muchtar., et al. conducted a Cochrane review of ten randomized controlled trials enrolling 2072 patients with APL 
and found that maintenance therapy compared to observation improved disease-free survival, but not overall survival [5]. Recent advances in APL therapy involving the incorporation of arsenic trioxide (ATO) into induction and consolidation regimens may eliminate the need for prolonged maintenance therapy, especially in low and intermediate risk APL. A phase 3 multicenter trial comparing ATRA plus chemotherapy with ATRA plus ATO in patients with low or intermediate risk APL demonstrated that ATRA plus ATO was not inferior to standard therapy, only the latter of which included maintenance chemotherapy [6]. PML should be considered in the context of maintenance chemotherapy for APL. Interruption of immunosuppressive drugs may lead to neurological recovery.

\section{Funding}

No funding was received for conceptualization and redaction of this manuscript.

\section{Authorship and Conflict-of-Interest Statements}

Sita Bhella and Catherine Maurice (first and second authors) have contributed equally to this manuscript.

Sita Bhella has a consultancy role to disclose with Lundbeck. She contributed to the conception, redaction and editing of this manuscript.

Catherine Maurice has no disclosure. She contributed to the conception, redaction and editing of this manuscript.

Lili-Naz Hazrati has no disclosure. She contributed by her expertise in Neuro-Pathology to interpret the biopsy results leading to the diagnosis. She provided the pathology pictures and collaborated to the editing of the manuscript.

Robert Chen received honoraria from Merz and Allergan and has a consultancy role with Merz. He received funding not associated with this case report from Medtronic, Inc and Merz. He collaborated by his expert opinion in the editing of the manuscript.

Andre C. Schuh has a consultancy role to disclose with Amgen, Celgen and Lundbeck. He also provided an expert testimony on behalf of Lundbeck. He collaborated to the redaction and editing of the manuscript.

Matthew Seftel received honoraria from Lundbeck and has a consultancy role with Lundbeck. He collaborated to the conception and editing of this manuscript.
Warren P. Mason, Austin M. Pereira and Amanda Pereira have no disclosure and collaborated to the editing of the manuscript.

\section{Bibliography}

1. Tan CS and Koralnik IJ. "Progressive multifocal leukoencephalopathy and other disorders caused by JC virus: clinical features and pathogenesis". The Lancet Neurology 9 (2010): 425437.

2. Tan K., et al. "PML-IRIS in patients with HIV infection: clinical manifestations and treatment with steroids". Neurology 72 (2009): 1458-1464.

3. Di Pauli F., et al. "Progressive leukoencephalopathy complicating untreated chronic lymphatic leukemia: a case report and review of the literature". Journal of Clinical Virology 60.4 (2014): 424-427.

4. Weber T., et al. "Polymerase chain reaction for the detection of JC virus DNA in cerebrospinal fluid: a quality control study. European Union Concerted Action on Viral Meningitis and Encephalitis". Journal of Virology Methods 69.1-2 (1997): 231237.

5. Muchtar E., et al. "The role of maintenance therapy in acute promyelocytic leukemia in the first complete remission". Cochrane Database Systemic Reviews (2013).

6. Lo-Coco F., et al. "Retinoic acid and arsenic trioxide for acute promyelocytic leukemia". New England Journal of Medicine 369.2 (2013): 111-121.

\section{Assets from publication with us}

- Prompt Acknowledgement after receiving the article

- Thorough Double blinded peer review

- Rapid Publication

- Issue of Publication Certificate

- High visibility of your Published work

Website: https://www.actascientific.com/

Submit Article: https://www.actascientific.com/submission.php Email us: editor@actascientific.com

Contact us: +919182824667 